\title{
FUSION RULES AND COMPLETE REDUCIBILITY OF CERTAIN MODULES FOR AFFINE LIE ALGEBRAS
}

\author{
DRAŽEN ADAMOVIĆ AND OZREN PERŠE
}

\begin{abstract}
We develop a new method for obtaining branching rules for affine Kac-Moody Lie algebras at negative integer levels. This method uses fusion rules for vertex operator algebras of affine type. We prove that an infinite family of ordinary modules for affine vertex algebra of type $A$ investigated in AP1 is closed under fusion. Then we apply these fusion rules on explicit bosonic realization of level -1 modules for the affine Lie algebra of type $A_{\ell-1}^{(1)}$, obtain a new proof of complete reducibility for these representations, and the corresponding decomposition for $\ell \geq 3$. We also obtain the complete reducibility of the associated level -1 modules for affine Lie algebra of type $C_{\ell}^{(1)}$. Next we notice that the category of $D_{2 \ell-1}^{(1)}$ modules at level $-2 \ell+3$ obtained in P1 has the isomorphic fusion algebra. This enables us to decompose certain $E_{6}^{(1)}$ and $F_{4}^{(1)}$-modules at negative levels.
\end{abstract}

\section{INTRODUCTION}

In the present paper we study branching rules for certain modules for affine Lie algebras at negative levels. We study the free field realizations of modules for affine Lie algebra of type $A$ of level -1 introduced in $[\mathrm{FF}$ ] and certain representations which naturally appear in the context of conformal embeddings in our recent paper [AP4].

V. Kac and M. Wakimoto considered in [KW] the free field representations of the affine Lie superalgebra $\widehat{\mathfrak{g l}(m \mid \ell)}$ realized on the tensor product $F_{m} \otimes M_{\ell}$, where $F_{m}$ (resp. $M_{\ell}$ ) is a vertex algebra associated to infinitedimensional Clifford (resp. Weyl) algebra. They proved that every charge component $\left(F_{m} \otimes M_{\ell}\right)^{(s)}$ is an irreducible $\widehat{\mathfrak{g l}(m \mid \ell)}$-module if $m \geq 1$. Their proof can not be applied in the case $m=0$ since it uses super boson-fermion correspondence. Such approach requires at least one fermionic field, and it is a generalization of super boson-fermion correspondence based on $\widehat{\mathfrak{g l}(1 \mid 1)}$ from [KL]. So for the case $m=0$, one needs different approach. V. Kac and M. Wakimoto also noted in Remark 3.3. of [KW] that $M_{2}^{(s)}$ is not irreducible $\widehat{\mathfrak{g l}(2)}$-module. So this makes the problem of irreducibility of $M_{\ell}^{(s)}$ open for $\ell \geq 3$.

2000 Mathematics Subject Classification. Primary 17B69, Secondary 17B67, 81R10. 
In this paper we extend the Kac-Wakimoto irreducibility result to $\widehat{\mathfrak{g l}(0, \ell)}=$ $\widehat{\mathfrak{g l}(\ell)}$-modules $M_{\ell}^{(s)}$ for $\ell \geq 3$. We will prove that every charge component $M_{\ell}^{(s)}$ is an irreducible $\widehat{\mathfrak{g l}(\ell)}$-module.

Let us describe our construction in more detail.

Vertex operator algebra $M_{\ell}$ is simple and admits the charge decomposition $M_{\ell}=\oplus_{s \in \mathbb{Z}} M_{\ell}^{(s)}$. The charge zero subspace $M_{\ell}^{(0)}$ is a simple vertex operator subalgebra of $M_{\ell}$ which contains the vertex operator algebra $\widetilde{L}_{A_{\ell-1}^{(1)}}\left(-\Lambda_{0}\right)$ (defined by relation (2.4)) associated to affine Lie algebra of type $A_{\ell-1}^{(1)}$ of level -1 , and a copy of rank one Heisenberg vertex algebra $M(1)$, commuting with $\widetilde{L}_{A_{\ell-1}^{(1)}}\left(-\Lambda_{0}\right)$.

Let $\ell \geq 3$. In this paper we present a vertex algebraic proof of the isomorphism

$$
M_{\ell}^{(0)} \cong \widetilde{L}_{A_{\ell-1}^{(1)}}\left(-\Lambda_{0}\right) \otimes M(1)
$$

which proves that $\widetilde{L}_{A_{\ell-1}^{(1)}}\left(-\Lambda_{0}\right)$ is simple.

Our proof is based the classification results from [AP1], the notion of fusion rules for vertex algebra modules and the fact that $M_{\ell}$ is a simple vertex operator algebra. It turns out that $A_{\ell-1}^{(1)}$-modules which are realized inside of $M_{\ell}$ are modules for the vertex operator algebra $V_{\ell-1,1}$ investigated in [AP1], which enables us to prove that these modules are closed under fusion. Then explicit information on fusion rules and standard techniques in the vertex algebra theory prove (1.1). We also show that every $M_{\ell}^{(s)}$ $(s \in \mathbb{Z})$ is an irreducible $L_{A_{\ell-1}^{(1)}}\left(-\Lambda_{0}\right) \otimes M(1)$-module.

It is interesting to notice that (1.1) does not hold if $\ell \leq 2$. For $\ell=1$, $M_{\ell}^{(0)}$ is isomorphic to the vertex algebra $\mathcal{W}_{1+\infty}$ at central charge $c=-1$ (cf. [KR], A2, [L], W]). The case $\ell=2$ was described in [KW].

Next, we consider some vertex subalgebras of $L_{A_{2 \ell-1}^{(1)}}\left(-\Lambda_{0}\right)$ of affine type. In this paper we shall consider the vertex subalgebra of type $C$, and the associated bosonic representation $M_{2 \ell}$. The results from [AP3] then imply that $M_{2 \ell}$ is a completely reducible $L_{C_{\ell}^{(1)}}\left(-\Lambda_{0}\right)$-module.

In this paper we also find that there can exist vertex operator algebras of affine type having the same fusion algebras as $L_{A_{\ell-1}^{(1)}}\left(-\Lambda_{0}\right)$. For that purpose, we study fusion rules for vertex operator algebras of type $D$ introduced recently in [P1]. We are focused on the category of ordinary modules for vertex operator algebra $\mathcal{V}_{D_{2 \ell-1}^{(1)}}\left(-(2 \ell-3) \Lambda_{0}\right)$ which is a non-trivial quotient of the universal vertex operator algebra of level $-2 \ell+3$. In order to determine the fusion rules, one needs to decompose certain tensor products of irreducible finite-dimensional representations of type $D$. But this job was 
done by S. Okada in $[\mathrm{O}$. The required tensor product is multiplicity free, and by using his result we are able to calculate fusion rules for the irreducible representations classified in $\mathrm{P} 1$. These fusion rules have many interesting applications.

We first classify irreducible representations for the simple vertex operator algebra $L_{D_{2 \ell-1}^{(1)}}\left(-(2 \ell-3) \Lambda_{0}\right)$. Next we are focused on the case $\ell=3$. Then the simple vertex operator algebra $L_{D_{5}^{(1)}}\left(-3 \Lambda_{0}\right)$ can be realized as a subalgebra of the simple vertex operator algebra $L_{E_{6}^{(1)}}\left(-3 \Lambda_{0}\right)$. By combining our fusion rules analysis, we are able to decompose the vertex operator algebra $L_{E_{6}^{(1)}}\left(-3 \Lambda_{0}\right)$ as a direct sum of irreducible $L_{D_{5}^{(1)}}\left(-3 \Lambda_{0}\right) \otimes M(1)$-modules, where $M(1)$ is the Heisenberg vertex operator algebra with central charge 1. We obtain certain similar decompositions which follow from the results on conformal embeddings from [AP4.

We assume that the reader is familiar with the notion of vertex operator algebra (cf. [B], FHL, [FLM], [FB], FZ], [K2], Li], [LL) and Kac-Moody algebra (cf. [K1]). Throughout the paper, we use the following notation: $\mathfrak{g}_{X_{\ell}}$ for simple Lie algebra of type $X_{\ell}$, and $\hat{\mathfrak{g}}_{X_{\ell}}$ for the associated affine Lie algebra of type $X_{\ell}^{(1)}, V_{X_{\ell}}(\mu)$ for irreducible highest weight module for $\mathfrak{g}_{X_{\ell}}$, $L_{X_{\ell}^{(1)}}(\lambda)$ for irreducible highest weight module for $\hat{\mathfrak{g}}_{X_{\ell}}$ and $N_{X_{\ell}^{(1)}}\left(k \Lambda_{0}\right)$ for the universal affine vertex algebra of level $k$ associated to $\hat{\mathfrak{g}}_{X_{\ell}}$.

Acknowledgments: We would like to thank the referee for his valuable comments.

\section{Weyl Vertex ALGEBRAS AND some AFFine Lie ALGEBRAS}

The Weyl algebra $W_{\ell}\left(\frac{1}{2}+\mathbb{Z}\right)$ is a complex associative algebra generated by

$$
a_{i}^{ \pm}(r), \quad r \in \frac{1}{2}+\mathbb{Z}, 1 \leq i \leq \ell
$$

with non-trivial relations

$$
\left[a_{i}^{+}(r), a_{j}^{-}(s)\right]=\delta_{r+s, 0} \delta_{i, j}
$$

where $r, s \in \frac{1}{2}+\mathbb{Z}, i, j \in\{1, \ldots, \ell\}$.

Let $M_{\ell}$ be the irreducible $W_{\ell}\left(\frac{1}{2}+\mathbb{Z}\right)$-module generated by the cyclic vector 1 such that

$$
a_{i}^{ \pm}(r) \mathbf{1}=0 \text { for } r>0,1 \leq i \leq \ell .
$$

Define the following fields on $M_{\ell}$

$$
a_{i}^{ \pm}(z)=\sum_{n \in \mathbb{Z}} a_{i}^{ \pm}\left(n+\frac{1}{2}\right) z^{-n-1}, \quad 1 \leq i \leq \ell .
$$


The fields $a_{i}^{ \pm}(z), i=1, \ldots, \ell$ generate on $M_{\ell}$ the unique structure of a simple vertex algebra (cf. [K2], $[\mathrm{FB}]$ ). Let us denote the corresponding vertex operator by $Y$.

We have the following conformal vector in $M_{\ell}$ :

$$
\omega=\frac{1}{2} \sum_{i=1}^{\ell}\left(a_{i}^{-}\left(-\frac{3}{2}\right) a_{i}^{+}\left(-\frac{1}{2}\right)-a_{i}^{+}\left(-\frac{3}{2}\right) a_{i}^{-}\left(-\frac{1}{2}\right)\right) \mathbf{1} .
$$

Let $Y(\omega, z)=\sum_{n \in \mathbb{Z}} L(n) z^{-n-2}$. Then $M_{\ell}$ is a $\frac{1}{2} \mathbb{Z}_{\geq 0}$-graded with respect to $L(0)$ :

$$
M_{\ell}=\bigoplus_{m \in \frac{1}{2} \mathbb{Z}_{\geq 0}} M_{\ell}(m), \quad M_{\ell}(m)=\left\{v \in M_{\ell} \mid L(0) v=m v\right\} .
$$

Note that $M_{\ell}(0)=\mathbb{C} 1$. For $v \in M_{\ell}(m)$ we shall write $\operatorname{wt}(v)=m$.

Define

$$
H=-\sum_{i=1}^{\ell} a_{i}^{+}\left(-\frac{1}{2}\right) a_{i}^{-}\left(-\frac{1}{2}\right) \mathbf{1} .
$$

Let $M(1)$ be the Heisenberg vertex subalgebra of $M_{\ell}$ generated by $H$. Let $Y(H, z)=\sum_{n \in \mathbb{Z}} H(n) z^{-n-1}$.

Then

$$
\left[H(0), a_{i}^{ \pm}(n)\right]= \pm a_{i}^{ \pm}(n), \quad 1 \leq i \leq \ell .
$$

Clearly, $H(0)$ acts semisimply on $M_{\ell}$ and it defines the following $\mathbb{Z}$-gradation, called the charge decomposition:

$$
M_{\ell}=\bigoplus_{s \in \mathbb{Z}} M_{\ell}^{(s)}, \quad M_{\ell}^{(s)}=\left\{v \in M_{\ell} \mid H(0) v=s v\right\} .
$$

Denote by $M(1, r)$ the irreducible $M(1)$-module on which $H(0)$ acts as $r \mathrm{Id}$, $r \in \mathbb{C}$.

The subalgebra of $M_{\ell}$ generated by

$$
\begin{aligned}
& e_{\epsilon_{i}-\epsilon_{j}}=a_{i}^{+}\left(-\frac{1}{2}\right) a_{j}^{-}\left(-\frac{1}{2}\right) \mathbf{1}, \quad f_{\epsilon_{i}-\epsilon_{j}}=a_{i}^{-}\left(-\frac{1}{2}\right) a_{j}^{+}\left(-\frac{1}{2}\right) \mathbf{1}, \\
& h_{\epsilon_{i}-\epsilon_{j}}=-a_{i}^{+}\left(-\frac{1}{2}\right) a_{i}^{-}\left(-\frac{1}{2}\right) \mathbf{1}+a_{j}^{+}\left(-\frac{1}{2}\right) a_{j}^{-}\left(-\frac{1}{2}\right) \mathbf{1},
\end{aligned}
$$

$$
\text { for } i, j=1, \ldots, \ell, i<j,
$$

is level -1 affine vertex operator algebra associated to the affine Lie algebra $\hat{\mathfrak{g}}_{A_{\ell-1}}$ of type $A_{\ell-1}^{(1)}$. By universal property of the vertex algebra $N_{A_{\ell-1}^{(1)}}\left(-\Lambda_{0}\right)$ (cf. [K2]), we get a homomorphism of vertex algebras

$$
\Phi: N_{A_{\ell-1}^{(1)}}\left(-\Lambda_{0}\right) \rightarrow M_{\ell}^{0} .
$$

The image of this homomorphism we denote by $\widetilde{L}_{A_{\ell-1}^{(1)}}\left(-\Lambda_{0}\right)$, i.e.

$$
\widetilde{L}_{A_{\ell-1}^{(1)}}\left(-\Lambda_{0}\right)=\operatorname{Im}(\Phi) .
$$


Claim of the following proposition was given in the proof of Proposition 5 from [AP3 (see also Proposition 2 from that paper) in the case when $\ell$ is even. The proof in the case when $\ell$ is odd is analogous.

Proposition 2.1. AAP3] Vertex subalgebra $\widetilde{L}_{A_{\ell-1}^{(1)}}\left(-\Lambda_{0}\right) \otimes M(1)$ of $M_{\ell}$ has the same conformal vector as $M_{\ell}$. Thus

$$
\omega=\omega_{\text {Sug }}+\omega_{1} \text {, }
$$

where $\omega_{\text {Sug }}$ is conformal vector in $\widetilde{L}_{A_{\ell-1}^{(1)}}\left(-\Lambda_{0}\right)$ obtained by the Sugawara construction and

$$
\omega_{1}=-\frac{1}{2 \ell} H(-1)^{2} \mathbf{1}
$$

is a conformal vector in $M(1)$.

Proof. We will briefly sketch the proof, and omit details because of its similarity with the proof of Proposition 2 from [AP3. Let us denote

$H^{(i)}=-\sum_{r=1}^{i} a_{r}^{+}\left(-\frac{1}{2}\right) a_{r}^{-}\left(-\frac{1}{2}\right) \mathbf{1}+i a_{i+1}^{+}\left(-\frac{1}{2}\right) a_{i+1}^{-}\left(-\frac{1}{2}\right) \mathbf{1}, \quad$ for $i=1, \ldots, \ell-1$.

Using explicit formula for Sugawara conformal vector (cf. [FZ], [Li], [FB], [K2], [LL]), we obtain:

$$
\begin{aligned}
\omega_{\text {Sug }}= & \frac{1}{2(\ell-1)}\left(\sum_{\substack{i, j=1 \\
i<j}}^{\ell}\left(e_{\epsilon_{i}-\epsilon_{j}}(-1) f_{\epsilon_{i}-\epsilon_{j}}(-1)+f_{\epsilon_{i}-\epsilon_{j}}(-1) e_{\epsilon_{i}-\epsilon_{j}}(-1)\right) \mathbf{1}\right. \\
& \left.+\sum_{i=1}^{\ell-1} \frac{1}{i(i+1)} H^{(i)}(-1)^{2} \mathbf{1}\right) .
\end{aligned}
$$

Using relations (2.3) we obtain

$$
\begin{aligned}
& \left(e_{\epsilon_{i}-\epsilon_{j}}(-1) f_{\epsilon_{i}-\epsilon_{j}}(-1)+f_{\epsilon_{i}-\epsilon_{j}}(-1) e_{\epsilon_{i}-\epsilon_{j}}(-1)\right) \mathbf{1} \\
& =2 a_{i}^{+}\left(-\frac{1}{2}\right) a_{i}^{-}\left(-\frac{1}{2}\right) a_{j}^{+}\left(-\frac{1}{2}\right) a_{j}^{-}\left(-\frac{1}{2}\right) \mathbf{1} \\
& +a_{i}^{-}\left(-\frac{3}{2}\right) a_{i}^{+}\left(-\frac{1}{2}\right) \mathbf{1}+a_{j}^{-}\left(-\frac{3}{2}\right) a_{j}^{+}\left(-\frac{1}{2}\right) \mathbf{1} \\
& -a_{i}^{+}\left(-\frac{3}{2}\right) a_{i}^{-}\left(-\frac{1}{2}\right) \mathbf{1}-a_{j}^{+}\left(-\frac{3}{2}\right) a_{j}^{-}\left(-\frac{1}{2}\right) \mathbf{1} \quad(i<j) .
\end{aligned}
$$

Now, direct calculations give:

$$
\omega_{\text {Sug }}=\frac{1}{2} \sum_{i=1}^{\ell}\left(a_{i}^{-}\left(-\frac{3}{2}\right) a_{i}^{+}\left(-\frac{1}{2}\right)-a_{i}^{+}\left(-\frac{3}{2}\right) a_{i}^{-}\left(-\frac{1}{2}\right)\right) \mathbf{1}+\frac{1}{2 \ell} H(-1)^{2} \mathbf{1},
$$

which implies the claim of proposition.

Proposition 2.1 implies that $\widetilde{L}_{A_{\ell-1}^{(1)}}\left(-\Lambda_{0}\right) \otimes M(1)$-submodule

$$
\widetilde{L}_{A_{\ell-1}^{(1)}}\left(-\Lambda_{0}+\mu\right) \otimes M(1, s)
$$


of $M_{\ell}$ has lowest conformal weight

$$
\frac{(\mu, \mu+2 \rho)}{2(\ell-1)}-\frac{s^{2}}{2 \ell}
$$

Modules $M_{\ell}^{(s)}$ admit the following $\mathbb{Z}_{\geq 0}$-gradation:

$$
M_{\ell}^{(s)}=\bigoplus_{n=0}^{\infty} M_{\ell}^{(s)}(n), \quad M_{\ell}^{(s)}(n)=\left\{v \in M_{\ell}^{(s)} \mid L(0) v=(|s| / 2+n) v\right\} .
$$

Lowest component of $M_{\ell}^{(s)}$ consists of vectors of weight $|s| / 2$ with respect to $L(0)$ and it is generated by $a_{1}^{+}(-1 / 2)^{s} \mathbf{1}$ if $s \geq 0$ and by $a_{\ell}^{-}(-1 / 2)^{-s} \mathbf{1}$ if $s<0$.

Lemma 2.1. We have:

$$
\widetilde{L}_{A_{\ell-1}^{(1)}}\left(-\Lambda_{0}\right) \otimes M(1) \cdot a_{1}^{+}(-1 / 2)^{s} \mathbf{1} \cong \widetilde{L}_{A_{\ell-1}^{(1)}}\left(-(s+1) \Lambda_{0}+s \Lambda_{1}\right) \otimes M(1, s),
$$

$\widetilde{L}_{A_{\ell-1}^{(1)}}\left(-\Lambda_{0}\right) \otimes M(1) \cdot a_{\ell}^{-}(-1 / 2)^{s} \mathbf{1} \cong \widetilde{L}_{A_{\ell-1}^{(1)}}\left(-(s+1) \Lambda_{0}+s \Lambda_{\ell-1}\right) \otimes M(1,-s)$, where $\widetilde{L}_{A_{\ell-1}^{(1)}}\left(-(s+1) \Lambda_{0}+s \Lambda_{1}\right)$ and $\widetilde{L}_{A_{\ell-1}^{(1)}}\left(-(s+1) \Lambda_{0}+s \Lambda_{\ell-1}\right)$ are certain highest weight $A_{\ell-1}^{(1)}$-modules with highest weights

$$
\lambda_{s}=-(s+1) \Lambda_{0}+s \Lambda_{1} \quad \text { and } \quad \mu_{s}=-(s+1) \Lambda_{0}+s \Lambda_{\ell-1} .
$$

Moreover, lowest components of these modules are finite-dimensional $\mathfrak{g}_{A_{\ell-1}}$ modules isomorphic to $V_{A_{\ell-1}}\left(s \omega_{1}\right)$ and $V_{A_{\ell-1}}\left(s \omega_{\ell-1}\right)$, respectively.

Proof. The proof follows from the fact that $a_{1}^{+}(-1 / 2)^{s} \mathbf{1}\left(\right.$ resp. $\left.a_{\ell}^{-}(-1 / 2)^{s} \mathbf{1}\right)$ is a singular vector for $\hat{\mathfrak{g}}_{A_{\ell-1}}$ of weight $-(s+1) \Lambda_{0}+s \Lambda_{1}\left(\operatorname{resp} .(s+1) \Lambda_{0}+\right.$ $\left.s \Lambda_{\ell-1}\right)$. Since $\mathfrak{g}_{A_{\ell-1}}$-submodule generated by this singular vector belongs to a lowest component of $M_{\ell}^{(s)}$, which is finite-dimensional, we get that this module must be irreducible.

\section{Main Results And Steps of the PRoOF}

The main result of the paper is that, for $\ell \geq 3$, every charge component $M_{\ell}^{(s)}(s \in \mathbb{Z})$ is an irreducible $\widehat{\mathfrak{g l}(\ell)}$-module. In this section we list the main steps of the proof of that claim.

The following proposition is standard (see, for example, Proposition 5.1 of [A4 for a similar version). For completeness we will also include this proof.

Proposition 3.1. $M_{\ell}^{(0)}$ is a simple vertex subalgebra of $M_{\ell}$. Each $M_{\ell}^{(s)}$ $(s \in \mathbb{Z})$ is a simple $M_{\ell}^{(0)}-$ module. 
Proof. Clearly, the charge zero component $M_{\ell}^{(0)}$ is a vertex subalgebra of $M$, and for every $r, s \in \mathbb{Z}$ we have that

$$
M_{\ell}^{(r)} \cdot M_{\ell}^{(s)}=\operatorname{span}_{\mathbb{C}}\left\{a_{j} b \mid a \in M_{\ell}^{(r)}, b \in M_{\ell}^{(s)}, j \in \mathbb{Z}\right\} \subset M_{\ell}^{(r+s)} .
$$

Let $v \in M_{\ell}^{(r)}$. Since $M_{\ell}$ is a simple vertex algebra, by Corollary 4.2 of [DM] we have that

$$
M_{\ell}=M_{\ell \cdot v}=\operatorname{span}_{\mathbb{C}}\left\{a_{j} v \mid a \in M_{\ell}, j \in \mathbb{Z}\right\} .
$$

This implies that $M_{\ell}^{(r)}=M_{\ell}^{(0)} \cdot v$, which proves simplicity of $M_{\ell}^{(r)}$ as $M_{\ell}^{(0)}$ module and simplicity of the vertex subalgebra $M_{\ell}^{(0)}$.

The crucial step in proof of the main result is the following theorem, which is also significant by itself:

Theorem 3.1. Assume that $\ell \geq 3$. We have:

$$
M_{\ell}^{(0)} \cong \widetilde{L}_{A_{\ell-1}^{(1)}}\left(-\Lambda_{0}\right) \otimes M(1) .
$$

In particular, $\widetilde{L}_{A_{\ell-1}^{(1)}}\left(-\Lambda_{0}\right)=U\left(\hat{\mathfrak{g}}_{A_{\ell-1}}\right) . \mathbf{1}$ is a simple vertex operator algebra.

The proof of Theorem 3.1 will use the classification result from [AP1], which we recall in Section 4, and certain fusion rules arguments. We present that proof in separate Section 5 .

Theorem 3.2. Assume that $\ell \geq 3$ and $s \in \mathbb{Z}$. Then $M_{\ell}^{(s)}$ is an irreducible $\widehat{\mathfrak{g l}(\ell)}-$ module. In particular

$$
\begin{array}{ll}
M_{\ell}^{(s)} \cong L_{A_{\ell-1}^{(1)}}\left(-(s+1) \Lambda_{0}+s \Lambda_{1}\right) \otimes M(1, s) & (s \geq 0), \\
M_{\ell}^{(s)} \cong L_{A_{\ell-1}^{(1)}}\left((s-1) \Lambda_{0}-s \Lambda_{\ell-1}\right) \otimes M(1, s) & (s<0) .
\end{array}
$$

Proof. Theorem 3.1 and Proposition 3.1 give that $M_{\ell}^{(s)}$ is an irreducible $L_{A_{\ell-1}^{(1)}}\left(-\Lambda_{0}\right) \otimes M(1)$-module. Now assertion follows from Lemma 2.1 .

\section{Affine vertex algebras of type $A$ and Level -1}

Our proof of main result (Theorem 3.1) will use the representation theory of the vertex algebra $\widetilde{L}_{A_{\ell-1}^{(1)}}\left(-\Lambda_{0}\right)$ realized as a subalgebra of the Weyl vertex algebra $M_{\ell}$. In this section we shall see that $\widetilde{L}_{A_{\ell-1}^{(1)}}\left(-\Lambda_{0}\right)$ is a certain quotient of the vertex algebra $V_{\ell-1,1}$ studied in [AP1]. Then the classification result for $V_{\ell-1,1}$ will automatically give the classification of irreducible $\widetilde{L}_{A_{\ell-1}^{(1)}}\left(-\Lambda_{0}\right)$-modules. (It is also possible that $\left.\widetilde{L}_{A_{\ell-1}^{(1)}}\left(-\Lambda_{0}\right) \cong V_{\ell-1,1}\right)$.

So we shall first recall some of our previous results from [AP1] on affine vertex algebras of type $A$ and level -1, and apply them to the present situation. 
In [AP1, we studied the following quotient of universal affine vertex algebra $N_{A_{\ell-1}^{(1)}}\left(-\Lambda_{0}\right)$, denoted by $V_{\ell-1,1}$ in that paper:

$$
V_{\ell-1,1}=\frac{N_{A_{\ell-1}^{(1)}}\left(-\Lambda_{0}\right)}{<v_{\ell-1,1}>}
$$

where $\left\langle v_{\ell-1,1}\right\rangle$ denotes the ideal generated by singular vector

$v_{\ell-1,1}=\left\{\begin{array}{cc}e_{\epsilon_{1}-\epsilon_{\ell}}(-1) e_{\epsilon_{2}-\epsilon_{\ell-1}}(-1) \mathbf{1}-e_{\epsilon_{2}-\epsilon_{\ell}}(-1) e_{\epsilon_{1}-\epsilon_{\ell-1}}(-1) \mathbf{1}, & \ell \geq 4 \\ e_{\epsilon_{2}-\epsilon_{3}}(-1)^{2} e_{\epsilon_{1}-\epsilon_{2}}(-1) \mathbf{1}-e_{\epsilon_{1}-\epsilon_{3}}(-1) e_{\epsilon_{2}-\epsilon_{3}}(-1) h_{\epsilon_{1}-\epsilon_{2}}(-1) \mathbf{1} & \\ -e_{\epsilon_{1}-\epsilon_{3}}(-1)^{2} f_{\epsilon_{1}-\epsilon_{2}}(-1) \mathbf{1}, & \ell=3\end{array}\right.$ in $N_{A_{\ell-1}^{(1)}}\left(-\Lambda_{0}\right)$.

Using the method for classification of representations of affine vertex algebras based on singular vectors (from [A1], $\mathrm{AM}], \mathrm{MP}]$ ), we obtained the following result (see Theorem 5.7 and Corollary 6.7 from [AP1]):

Proposition 4.1. AP1 Let $\ell \geq 3$. The set

$$
\left\{L_{A_{\ell-1}^{(1)}}\left(-(s+1) \Lambda_{0}+s \Lambda_{1}\right), L_{A_{\ell-1}^{(1)}}\left(-(s+1) \Lambda_{0}+s \Lambda_{\ell-1}\right) \mid s \in \mathbb{Z}_{\geq 0}\right\}
$$

provides a complete list of irreducible ordinary $V_{\ell-1,1}-$ modules.

The following result is a modification of Proposition 4.1 which we need in this paper.

Proposition 4.2. Let $\ell \geq 3$.

(i) There is a surjective homomorphism of vertex algebras

$$
\widetilde{\Phi}: V_{\ell-1,1} \rightarrow \widetilde{L}_{A_{\ell-1}^{(1)}}\left(-\Lambda_{0}\right) .
$$

(ii) The set

$$
\left\{L_{A_{\ell-1}^{(1)}}\left(-(s+1) \Lambda_{0}+s \Lambda_{1}\right), L_{A_{\ell-1}^{(1)}}\left(-(s+1) \Lambda_{0}+s \Lambda_{\ell-1}\right) \mid s \in \mathbb{Z}_{\geq 0}\right\}
$$

provides a complete list of irreducible ordinary $\widetilde{L}_{A_{\ell-1}^{(1)}}\left(-\Lambda_{0}\right)-$ modules.

Proof. Using homomorphism $\Phi: N_{A_{\ell-1}^{(1)}}\left(-\Lambda_{0}\right) \rightarrow \widetilde{L}_{A_{\ell-1}^{(1)}}\left(-\Lambda_{0}\right)$ defined in Section 2 and formulas for singular vectors $v_{\ell-1,1}$ one can easily verify that $\Phi\left(v_{\ell-1,1}\right)=0$ in $M_{\ell}$, for $\ell \geq 3$. This implies that

$$
\operatorname{Ker}(\Phi) \supset<v_{\ell-1,1}>
$$

and therefore we get homomorphism $\widetilde{\Phi}: V_{\ell-1,1} \rightarrow \widetilde{L}_{A_{\ell-1}^{(1)}}\left(-\Lambda_{0}\right)$.

Lemma 2.1 now implies that

$$
\widetilde{L}_{A_{\ell-1}^{(1)}}\left(-(s+1) \Lambda_{0}+s \Lambda_{1}\right), \widetilde{L}_{A_{\ell-1}^{(1)}}\left(-(s+1) \Lambda_{0}+s \Lambda_{\ell-1}\right) \quad\left(s \in \mathbb{Z}_{\geq 0}\right)
$$


are $\widetilde{L}_{A_{\ell-1}^{(1)}}\left(-\Lambda_{0}\right)$-modules, and therefore their simple quotients are also $\widetilde{L}_{A_{\ell-1}^{(1)}}\left(-\Lambda_{0}\right)-$ modules. Now assertion follows from Proposition 4.1 since every $\widetilde{L}_{A_{\ell-1}^{(1)}}\left(-\Lambda_{0}\right)-$ module is also a $V_{\ell-1,1}-$ module.

\section{Proof of Theorem 3.1}

In this section we give the proof of Theorem 3.1 .

The following lemma is a crucial technical result needed for the fusion rules argument used in the proof of Theorem 3.1 .

Lemma 5.1. Let $r, s \in \mathbb{Z}_{>0}, r \geq s$ and $\ell \geq 2$. Then the following tensor product decompositions hold:

(i) $\quad V_{A_{\ell}}\left(r \omega_{1}\right) \otimes V_{A_{\ell}}\left(s \omega_{1}\right)=$ $V_{A_{\ell}}\left((r+s) \omega_{1}\right) \oplus V_{A_{\ell}}\left((r+s-2) \omega_{1}+\omega_{2}\right) \oplus \cdots \oplus V_{A_{\ell}}\left((r-s) \omega_{1}+s \omega_{2}\right)$,

(ii) $\quad V_{A_{\ell}}\left(r \omega_{\ell}\right) \otimes V_{A_{\ell}}\left(s \omega_{\ell}\right)=$ $V_{A_{\ell}}\left((r+s) \omega_{\ell}\right) \oplus V_{A_{\ell}}\left((r+s-2) \omega_{\ell}+\omega_{\ell-1}\right) \oplus \cdots \oplus V_{A_{\ell}}\left((r-s) \omega_{\ell}+s \omega_{\ell-1}\right)$,

(iii) $\quad V_{A_{\ell}}\left(r \omega_{1}\right) \otimes V_{A_{\ell}}\left(s \omega_{\ell}\right)=$ $V_{A_{\ell}}\left((r-s) \omega_{1}\right) \oplus V_{A_{\ell}}\left((r-s+1) \omega_{1}+\omega_{\ell}\right) \oplus \cdots \oplus V_{A_{\ell}}\left(r \omega_{1}+s \omega_{\ell}\right)$.

Note that all weight subspaces of modules $V_{A_{\ell}}\left(r \omega_{1}\right)$ and $V_{A_{\ell}}\left(r \omega_{\ell}\right)$ are 1dimensional, and decompositions can be obtained by using Kostant formula [Ko. Decompositions of this type also appeared in [O].

\section{Proof of Theorem 3.1;}

First we notice that $M_{\ell}^{(0)}$ is completely reducible $M(1)$-module, isomorphic to an infinite direct sum of copies of $M(1)$.

Assume now that $\widetilde{L}_{A_{\ell-1}^{(1)}}\left(-\Lambda_{0}\right) \otimes M(1)$ is a proper submodule of $M_{\ell}^{(0)}$. Then we consider $M_{\ell}^{(0)}$ as a $\widetilde{L}_{A_{\ell-1}^{(1)}}\left(-\Lambda_{0}\right) \otimes M(1)$-module and conclude that there exists a singular vector $\Omega$ which is not proportional to 1 . Applying the classification result from Proposition 4.2 , we may assume that $\Omega$ has weight $\lambda_{r}$ or $\mu_{r}$ for certain $r \in \mathbb{Z}_{>0}$.

Assume first that $\Omega$ has weight $\lambda_{r}$. Then $U\left(\mathfrak{g}_{A_{\ell-1}}\right) . \Omega \cong V_{A_{\ell-1}}\left(r \omega_{1}\right)$.

Next we consider $M_{\ell}^{(0)}$-module $M_{\ell}^{(-r)}$ which is generated by vector $a_{\ell}^{-}(-1 / 2)^{r} \mathbf{1}$.

Since $\Omega$ and $a_{\ell}^{-}(-1 / 2)^{r} \mathbf{1}$ belong to the simple vertex algebra $M_{\ell}$, we conclude that

$$
Y(\Omega, z) a_{\ell}^{-}(-1 / 2)^{r} \mathbf{1} \neq 0
$$

Take $n_{0}$ such that

$$
\Omega_{n_{0}} a_{\ell}^{-}(-1 / 2)^{r} \mathbf{1} \neq 0, \quad \Omega_{n} a_{\ell}^{-}(-1 / 2)^{r} \mathbf{1}=0 \text { for } n>n_{0} .
$$


Then

$$
U=U\left(\mathfrak{g}_{A_{\ell-1}}\right) \cdot \Omega_{n_{0}} a_{\ell}^{-}(-1 / 2)^{r} \mathbf{1}
$$

is a $U\left(\mathfrak{g}_{A_{\ell-1}}\right)$-module which is the lowest component of $\widetilde{L}_{A_{\ell-1}^{(1)}}\left(-\Lambda_{0}\right)$-module $\widetilde{U}=U\left(\hat{\mathfrak{g}}_{A_{\ell-1}}\right) \cdot \Omega_{n_{0}} a_{\ell}^{-}(-1 / 2)^{r} \mathbf{1}$. Then Lemma 5.1 (iii) and the classification result from Proposition 4.2 give that $U$ is 1 -dimensional. Relation (2.5) now implies that $M_{\ell}^{(-r)}$ contains a non-trivial singular vector of conformal weight $-\frac{r^{2}}{2 \ell}$. This is a contradiction since $M_{\ell}^{(-r)}$ does not have vectors at this conformal weight (lowest conformal weight is $r / 2$ ).

Similarly we treat the case if $\Omega$ has weight $\mu_{r}$.

In this way we have proved that $M_{\ell}^{(0)} \cong \widetilde{L}_{A_{\ell-1}^{(1)}}\left(-\Lambda_{0}\right) \otimes M(1)$.

\section{Some APplications}

In this section we shall present some direct applications of the results from previous sections. We shall complete the classification process for simple affine vertex operator algebras $L_{A_{\ell-1}^{(1)}}\left(-\Lambda_{0}\right)$ and $L_{C_{\ell}^{(1)}}\left(-\Lambda_{0}\right)$ started in papers [A3], [AP1] and [AP3].

6.1. Fusion rules for $L_{A_{\ell-1}^{(1)}}\left(-\Lambda_{0}\right)$-modules. As a consequence of our construction and Proposition 4.2, we get the following classification result.

Theorem 6.1. Let $\ell \geq 3$. The set

$$
\left\{L_{A_{\ell-1}^{(1)}}\left(-(s+1) \Lambda_{0}+s \Lambda_{1}\right), L_{A_{\ell-1}^{(1)}}\left(-(s+1) \Lambda_{0}+s \Lambda_{\ell-1}\right) \mid s \in \mathbb{Z}_{\geq 0}\right\}
$$

provides a complete list of irreducible ordinary $L_{A_{\ell-1}^{(1)}}\left(-\Lambda_{0}\right)-$ modules.

We are also able to describe the fusion rules between irreducible $L_{A_{\ell-1}^{(1)}}\left(-\Lambda_{0}\right)-$ modules, for $\ell \geq 3$.

For every $s \in \mathbb{Z}$ we set $\pi_{s}= \begin{cases}L_{A_{\ell-1}^{(1)}}\left(-(s+1) \Lambda_{0}+s \Lambda_{1}\right) & \text { if } s \geq 0 \\ L_{A_{\ell-1}^{(1)}}\left((s-1) \Lambda_{0}-s \Lambda_{\ell-1}\right) & \text { if } s<0\end{cases}$

Next theorem will show that the fusion algebra generated by irreducible (ordinary) $L_{A_{\ell-1}^{(1)}}\left(-\Lambda_{0}\right)$-modules is isomorphic to the group algebra $\mathbb{C}[\mathbb{Z}]$.

Theorem 6.2. Assume that $i, j, k \in \mathbb{Z}$. The there exists a non-trivial intertwining operator of type

$$
\left(\begin{array}{cc}
\pi_{k} \\
\pi_{i} & \pi_{j}
\end{array}\right)
$$

if and only if $k=i+j$ 
Proof. Let $Y_{M_{\ell}}(\cdot, z)$ be the vertex operator map which defines on $M_{\ell}$ the structure of vertex operator algebra. Required non-trivial intertwining operators are realized by restriction, as follows:

$$
I(u, z) v=Y_{M_{\ell}}(u, z) v \quad\left(u \in M_{\ell}^{(i)}, v \in M_{\ell}^{(j)}\right) .
$$

The rest of statement easily follows from Lemma 5.1 by using standard fusion rules arguments.

Remark 6.1. Vertex operator algebra $L_{A_{\ell-1}^{(1)}}\left(-\Lambda_{0}\right)$ is not rational in the category $\mathcal{O}$, unlike admissible affine vertex operator algebras (cf. [A1], AM], [Ara], [P2], [P3]). Modules constructed in Remark 5.8 of [AP1] are examples of $L_{A_{\ell-1}^{(1)}}\left(-\Lambda_{0}\right)-$ modules from category $\mathcal{O}$ that are not completely reducible.

6.2. $M_{2 \ell}$ as a $C_{\ell}^{(1)}$-module. Next consequence of the construction from Section 5 is the complete reducibility of $M_{2 \ell}$ as a module for affine Lie algebra $C_{\ell}^{(1)}$.

By using conformal embedding of $L_{C_{\ell}^{(1)}}\left(-\Lambda_{0}\right)$ into $L_{A_{2 \ell-1}^{(1)}}\left(-\Lambda_{0}\right)$, the classification results from [A3], AP3] and Proposition 7 from [AP3] we get:

\section{Corollary 6.1.}

(i) Let $\ell \geq 3$. The set

$$
\left\{L_{C_{\ell}^{(1)}}\left(-(s+1) \Lambda_{0}+s \Lambda_{1}\right) \mid s \in \mathbb{Z}_{\geq 0}\right\} \cup\left\{L_{C_{\ell}^{(1)}}\left(-2 \Lambda_{0}+\Lambda_{2}\right)\right\}
$$

provides a complete list of irreducible ordinary $L_{C_{\ell}^{(1)}}\left(-\Lambda_{0}\right)$-modules.

(ii) $M_{2 \ell}$ is a completely reducible $L_{C_{\ell}^{(1)}}\left(-\Lambda_{0}\right)$-module.

6.3. On $\mathcal{W}_{1+\infty}$-algebra. Let $\mathcal{W}_{1+\infty,-\ell}$ denote the simple $\mathcal{W}_{1+\infty}$ vertex algebra with central charge $c=-\ell$. Then (cf. [KR])

$$
\mathcal{W}_{1+\infty,-\ell}=M_{\ell}^{\mathfrak{g l}(\ell)}
$$

Our results show that

$$
\mathcal{W}_{1+\infty,-\ell}=\left(L_{A_{\ell-1}^{(1)}}\left(-\Lambda_{0}\right) \otimes M(1)\right)^{\mathfrak{g l}(\ell)} \cong L_{A_{\ell-1}^{(1)}}\left(-\Lambda_{0}\right)^{\mathfrak{s l}(\ell)} \otimes M(1)
$$

if $\ell \geq 3$. (For $\ell \leq 2$ this is not true).

Therefore, $\mathcal{W}_{1+\infty,-\ell}$ is an affine orbifold.

Remark 6.2. As far as we understand, the above result is new in the mathematical literature. Note also that this result is in complete agreement with result from [FKRW]:

$$
\mathcal{W}_{1+\infty, \ell}=\left(L_{A_{\ell-1}^{(1)}}\left(\Lambda_{0}\right) \otimes M(1)\right)^{\mathfrak{g l}(\ell)} \cong L_{A_{\ell-1}^{(1)}}\left(\Lambda_{0}\right)^{\mathfrak{s l}(\ell)} \otimes M(1) .
$$




\section{Fusion Algebra for Certain $D_{2 \ell-1}^{(1)}$-MOdules of NEGAtive LEVELS}

Let $N_{D_{\ell}^{(1)}}\left(k \Lambda_{0}\right)$ be the universal affine vertex algebra of level $k$ associated to the affine Lie algebra of type $D_{\ell}^{(1)}$. It was proved in [P1] that the vector

$$
v=\sum_{i=2}^{\ell} e_{\epsilon_{1}-\epsilon_{i}}(-1) e_{\epsilon_{1}+\epsilon_{i}}(-1) \mathbf{1}
$$

is a singular vector in $N_{D_{\ell}^{(1)}}\left((-\ell+2) \Lambda_{0}\right)$. The classification of representations of vertex operator algebra

$$
\mathcal{V}_{D_{\ell}^{(1)}}\left((-\ell+2) \Lambda_{0}\right)=\frac{N_{D_{\ell}^{(1)}}\left((-\ell+2) \Lambda_{0}\right)}{<v>}
$$

where $\langle v\rangle$ is the ideal generated by singular vector $v$, was studied in that paper. We will recall the classification of all irreducible ordinary modules for $\mathcal{V}_{D_{\ell}^{(1)}}\left((-\ell+2) \Lambda_{0}\right)$.

It follows from P1 that:

Proposition 7.1. The set

$$
\left\{L_{D_{\ell}^{(1)}}\left(-(s+\ell-2) \Lambda_{0}+s \Lambda_{\ell-1}\right), L_{D_{\ell}^{(1)}}\left(-(s+\ell-2) \Lambda_{0}+s \Lambda_{\ell}\right) \mid s \in \mathbb{Z}_{\geq 0}\right\}
$$

gives all irreducible ordinary $\mathcal{V}_{D_{\ell}^{(1)}}\left((-\ell+2) \Lambda_{0}\right)$-modules.

In this section we shall prove that the above modules actually give all irreducible modules for the simple vertex operator algebra $L_{D_{\ell}^{(1)}}\left((-\ell+2) \Lambda_{0}\right)$ in the case when $\ell$ is odd.

Top components of irreducible modules from Proposition 7.1 are irreducible modules for the simple Lie algebra of type $D$. So we get an interesting series of modules:

$$
V_{D_{\ell}}\left(s \omega_{\ell-1}\right), V_{D_{\ell}}\left(s \omega_{\ell}\right) \quad\left(s \in \mathbb{Z}_{\geq 0}\right) .
$$

Let

$$
U_{D_{\ell}}(s):= \begin{cases}V_{D_{\ell}}\left(s \omega_{\ell-1}\right), & \text { for } s \geq 0 \\ V_{D_{\ell}}\left(-s \omega_{\ell}\right), & \text { for } s<0 .\end{cases}
$$

These modules appear in the representation theory of simple Lie algebras. Particulary important result is obtained by S. Okada [O]. He described characters of these representations and their tensor products. He proved that their tensor product is multiplicity free, i.e., every irreducible component has multiplicity one.

By using Theorem 2.5 of $[\mathrm{O}$ ] we have:

Proposition 7.2. Assume that $\ell \geq 3$ is an odd natural number. Assume that $r, s \in \mathbb{Z}$. Then $U_{D_{\ell}}(t)$ appears in the tensor product $U_{D_{\ell}}(r) \otimes U_{D_{\ell}}(s)$ if and only if $t=r+s$. The multiplicity is one. 
Proof. We present the proof in the case $r \geq 0$ and $s \geq 0$. Other cases are treated similarly. The combinatorial description of tensor product decomposition from Theorem 2.5 (3) (for $\ell$ odd) of [O] gives that

$$
V_{D_{\ell}}\left(r \omega_{\ell-1}\right) \otimes V_{D_{\ell}}\left(s \omega_{\ell-1}\right)=\bigoplus_{\mu} V_{D_{\ell}}(\mu),
$$

where the summation goes over dominant integral weights $\mu$ such that

$$
\mu=\left(k_{1} \omega_{1}+k_{3} \omega_{3}+k_{5} \omega_{5}+\ldots+k_{\ell-2} \omega_{\ell-2}\right)+k_{\ell-1} \omega_{\ell-1} \quad\left(k_{i} \in \mathbb{Z}_{\geq 0}\right),
$$

and

$$
2\left(k_{1}+k_{3}+\ldots+k_{\ell-2}\right)+k_{\ell-1}=r+s .
$$

This implies that modules $V_{D_{\ell}}\left(t \omega_{\ell}\right)$ do not appear in that tensor product and that $V_{D_{\ell}}\left(t \omega_{\ell-1}\right)$ appears only for $t=r+s$ (with multiplicity one). The claim follows.

This tensor product decomposition implies the following result about fusion rules:

Theorem 7.1. Assume that $\widetilde{\pi_{r}}, r \in \mathbb{Z}$ are $\mathbb{Z}_{\geq 0}$-graded $\mathcal{V}_{D_{2 \ell-1}^{(1)}}\left((-2 \ell+3) \Lambda_{0}\right)$ modules such that top component of $\widetilde{\pi}_{r}$ is isomorphic to the irreducible $\mathfrak{g}_{D_{2 \ell-1}}$-module $U_{D_{2 \ell-1}}(r)$. Let $\pi_{r}$ denote the associated simple quotient. Assume that there is a non-trivial intertwining operator of type

$$
\left(\begin{array}{cc}
\pi_{t} \\
\widetilde{\pi_{r}} & \pi_{s}
\end{array}\right)
$$

Then $t=r+s$.

Remark 7.1. It is interesting that in the case of $\mathcal{V}_{D_{2 \ell}^{(1)}}\left((-2 \ell+2) \Lambda_{0}\right)$-modules we get a different fusion algebra.

Now we shall apply our result on fusion rules to get complete classification of irreducible ordinary $L_{D_{2 \ell-1}^{(1)}}\left(-(2 \ell-3) \Lambda_{0}\right)$-modules.

Theorem 7.2. The set

$$
\left\{L_{D_{2 \ell-1}^{(1)}}\left(-(s+2 \ell-3) \Lambda_{0}+s \Lambda_{2 \ell-2}\right), L_{D_{2 \ell-1}^{(1)}}\left(-(s+2 \ell-3) \Lambda_{0}+s \Lambda_{2 \ell-1}\right) \mid s \in \mathbb{Z}_{\geq 0}\right\}
$$

provides the complete list of irreducible ordinary $L_{D_{2 \ell-1}^{(1)}}\left(-(2 \ell-3) \Lambda_{0}\right)-$ modules.

Proof. From Proposition 7.1 we get that every irreducible module for the simple vertex operator algebra $L_{D_{2 \ell-1}^{(1)}}\left(-(2 \ell-3) \Lambda_{0}\right)$ belongs to the set (7.7). It remains to prove that every module $\left(M, Y_{M}(\cdot, z)\right)$ from the set (17.7) is in fact a module for $L_{D_{2 \ell-1}^{(1)}}\left(-(2 \ell-3) \Lambda_{0}\right)$. 
Let $J\left(-(2 \ell-3) \Lambda_{0}\right)$ be the maximal ideal in the vertex operator algebra $\mathcal{V}_{D_{2 \ell-1}^{(1)}}\left(-(2 \ell-3) \Lambda_{0}\right)$. Since $J\left(-(2 \ell-3) \Lambda_{0}\right)$ is an ordinary $\mathcal{V}_{D_{2 \ell-1}^{(1)}}(-(2 \ell-$ 3) $\left.\Lambda_{0}\right)$-module, we conclude that it is generated by singular vectors which have weights $\lambda_{r}=-(r+2 \ell-3) \Lambda_{0}+r \Lambda_{2 \ell-2}$ or $\mu_{r}=-(r+2 \ell-3) \Lambda_{0}+r \Lambda_{2 \ell-1}$, $r \in \mathbb{Z}_{>0}$. Assume that there is a singular vector $\Omega$ of weight $\lambda_{r}$ or $\mu_{r}$ such that vertex operator $Y_{M}(\Omega, z)$ is non-zero on $M$. This leads to a non-trivial intertwining operator of type

$$
\left(\frac{M}{L\left(\lambda_{r}\right)} \quad M\right) \text { or }\left(\widetilde{M} \frac{M}{L\left(\mu_{r}\right)} M\right)
$$

This contradicts Theorem 7.1

Therefore, vertex operators $Y_{M}(v, z)$ must vanish for all $v \in J(-(2 \ell-$ $\left.3) \Lambda_{0}\right)$. This proves that $M$ is an $L_{D_{2 \ell-1}^{(1)}}\left(-(2 \ell-3) \Lambda_{0}\right)$-module.

\section{Conformal embedding of $L_{D_{5}^{(1)}}\left(-3 \Lambda_{0}\right) \otimes M(1)$ InTO $L_{E_{6}^{(1)}}\left(-3 \Lambda_{0}\right)$}

In this section we use the methods from Sections 3 and 5 and the fusion rules analysis from Section 7 to construct the conformal embedding of $L_{D_{5}^{(1)}}\left(-3 \Lambda_{0}\right) \otimes M(1)$ into $L_{E_{6}^{(1)}}\left(-3 \Lambda_{0}\right)$. We also determine the decomposition of $L_{E_{6}^{(1)}}\left(-3 \Lambda_{0}\right)$ into direct sum of $L_{D_{5}^{(1)}}\left(-3 \Lambda_{0}\right) \otimes M(1)$-modules.

Let $\mathfrak{g}_{E_{6}}$ be the simple Lie algebra of type $E_{6}$. We will use the construction of the root system of type $E_{6}$ from $[\mathrm{Bou}],[\mathrm{H}]$, and the notation for root vectors from AP4. Note now that the subalgebra of $\mathfrak{g}_{E_{6}}$ generated by (suitably chosen) positive root vectors

$$
\begin{aligned}
& e_{(5)}=e_{\frac{1}{2}\left(\epsilon_{8}-\epsilon_{7}-\epsilon_{6}+\epsilon_{5}-\epsilon_{4}-\epsilon_{3}-\epsilon_{2}-\epsilon_{1}\right)}, \\
& e_{\alpha_{2}}=e_{\epsilon_{2}+\epsilon_{1}}, \\
& e_{\alpha_{4}}=e_{\epsilon_{3}-\epsilon_{2}}, \\
& e_{\alpha_{3}}=e_{\epsilon_{2}-\epsilon_{1}}, \\
& e_{\alpha_{5}}=e_{\epsilon_{4}-\epsilon_{3}}
\end{aligned}
$$

and associated negative root vectors is a simple Lie algebra of type $D_{5}$. We denote this Lie algebra by $\mathfrak{g}_{D_{5}}$. Thus, $\mathfrak{g}_{E_{6}}$ has a reductive subalgebra $\mathfrak{g}_{D_{5}} \oplus \mathfrak{h}$, where $\mathfrak{h}=\mathbb{C} H$, and

$$
H=\frac{1}{3}\left(h_{8}-h_{7}-h_{6}-3 h_{5}\right)
$$

(where $h_{i}$ are determined by $\epsilon_{i}\left(h_{j}\right)=\delta_{i j}$ ), with the associated decomposition

$$
\mathfrak{g}_{E_{6}}=\mathfrak{g}_{D_{5}} \oplus \mathfrak{h} \oplus V_{D_{5}}\left(\omega_{4}\right) \otimes \mathbb{C}_{1} \oplus V_{D_{5}}\left(\omega_{5}\right) \otimes \mathbb{C}_{-1},
$$

where $\mathbb{C}_{r}$ denotes the one-dimensional $\mathfrak{h}$-module on which $H$ acts as scalar $r \in \mathbb{C}$. The highest weight vectors of $\mathfrak{g}_{D_{5}}$-modules $V_{D_{5}}\left(\omega_{4}\right)$ and $V_{D_{5}}\left(\omega_{5}\right)$ are $e_{(234)}$ and $e_{\epsilon_{5}+\epsilon_{4}}$, respectively. 
Denote by $\widetilde{L}_{D_{5}^{(1)}}\left(-3 \Lambda_{0}\right)$ the subalgebra of $L_{E_{6}^{(1)}}\left(-3 \Lambda_{0}\right)$ generated by $\mathfrak{g}_{D_{5}}$ and by $M(1)$ the Heisenberg vertex subalgebra of $L_{E_{6}^{(1)}}\left(-3 \Lambda_{0}\right)$ generated by $H$. Relation (8.8) and the criterion for conformal embeddings from [AP4] give:

Proposition 8.1. Vertex operator algebra $\widetilde{L}_{D_{5}^{(1)}}\left(-3 \Lambda_{0}\right) \otimes M(1)$ is conformally embedded in $L_{E_{6}^{(1)}}\left(-3 \Lambda_{0}\right)$.

As before, denote by $M(1, s)$ the irreducible $M(1)$-module on which $H(0)$ acts as scalar $s \in \mathbb{C}$. It follows that $\widetilde{L}_{D_{5}^{(1)}}\left(-3 \Lambda_{0}\right) \otimes M(1)$-submodule

$$
\widetilde{L}_{D_{5}^{(1)}}\left(-3 \Lambda_{0}+\mu\right) \otimes M(1, s)
$$

of $L_{E_{6}^{(1)}}\left(-3 \Lambda_{0}\right)$ has lowest conformal weight

$$
\frac{(\mu, \mu+2 \rho)}{10}-\frac{s^{2}}{8}
$$

It was shown in $\mathrm{AP} 4$ that

$$
\begin{aligned}
v_{E_{6}} & =\left(e_{(5)}(-1) e_{(12345)}(-1)+e_{(125)}(-1) e_{(345)}(-1)\right. \\
& \left.+e_{(135)}(-1) e_{(245)}(-1)+e_{(235)}(-1) e_{(145)}(-1)\right) \mathbf{1}
\end{aligned}
$$

is a singular vector in $N_{E_{6}^{(1)}}\left(-3 \Lambda_{0}\right)$. Thus, this vector is trivial in $L_{E_{6}^{(1)}}\left(-3 \Lambda_{0}\right)$.

The subalgebra of $N_{E_{6}^{(1)}}\left(-3 \Lambda_{0}\right)$ generated by $\mathfrak{g}_{D_{5}}$ is isomorphic to $N_{D_{5}^{(1)}}\left(-3 \Lambda_{0}\right)$. Note that $v_{E_{6}} \in N_{D_{5}^{(1)}}\left(-3 \Lambda_{0}\right)$, and that in fact $v_{E_{6}}$ is equal to vector $v$ from relation (7.6), for $\ell=5$. Since $v_{E_{6}}$ is trivial in $L_{E_{6}^{(1)}}\left(-3 \Lambda_{0}\right)$, we conclude that $\widetilde{L}_{D_{5}^{(1)}}\left(-3 \Lambda_{0}\right)$ is a quotient of vertex algebra $\mathcal{V}_{D_{5}^{(1)}}\left(-3 \Lambda_{0}\right)$ from Section 7 The classification results from Proposition 7.1 and Theorem 7.2 now give:

Proposition 8.2. The set

$$
\left\{L_{D_{5}^{(1)}}\left(-(s+3) \Lambda_{0}+s \Lambda_{4}\right), L_{D_{5}^{(1)}}\left(-(s+3) \Lambda_{0}+s \Lambda_{5}\right) \mid s \in \mathbb{Z}_{\geq 0}\right\}
$$

provides the complete list of irreducible ordinary $\widetilde{L}_{D_{5}^{(1)}}\left(-3 \Lambda_{0}\right)$-modules.

Vectors $e_{(234)}(-1)^{s} \mathbf{1}$ and $e_{\epsilon_{5}+\epsilon_{4}}(-1)^{s} \mathbf{1}$ are (non-trivial) singular vectors for $\hat{\mathfrak{g}}_{D_{5}}$ in $L_{E_{6}^{(1)}}\left(-3 \Lambda_{0}\right)$ of highest weights $-(s+3) \Lambda_{0}+s \Lambda_{4}$ and $-(s+3) \Lambda_{0}+$ $s \Lambda_{5}$, respectively. Furthermore, we have:

$$
\begin{aligned}
& {\left[H(0), e_{(234)}(n)\right]=e_{(234)}(n),} \\
& {\left[H(0), e_{\epsilon_{5}+\epsilon_{4}}(n)\right]=-e_{\epsilon_{5}+\epsilon_{4}}(n) .}
\end{aligned}
$$

We have:

$$
\widetilde{L}_{D_{5}^{(1)}}\left(-3 \Lambda_{0}\right) \otimes M(1) \cdot e_{(234)}(-1)^{s} \mathbf{1} \cong \widetilde{L}_{D_{5}^{(1)}}\left(-(s+3) \Lambda_{0}+s \Lambda_{4}\right) \otimes M(1, s),
$$


$\widetilde{L}_{D_{5}^{(1)}}\left(-3 \Lambda_{0}\right) \otimes M(1) \cdot e_{\epsilon_{5}+\epsilon_{4}}(-1)^{s} \mathbf{1} \cong \widetilde{L}_{D_{5}^{(1)}}\left(-(s+3) \Lambda_{0}+s \Lambda_{5}\right) \otimes M(1,-s)$, where $\widetilde{L}_{D_{5}^{(1)}}\left(-(s+3) \Lambda_{0}+s \Lambda_{4}\right)$ and $\widetilde{L}_{D_{5}^{(1)}}\left(-(s+3) \Lambda_{0}+s \Lambda_{5}\right)$ are certain highest weight $D_{5}^{(1)}$-modules with corresponding highest weights.

Clearly, $H(0)$ acts semisimply on $L_{E_{6}^{(1)}}\left(-3 \Lambda_{0}\right)$ and it defines the following $\mathbb{Z}$-gradation:

$L_{E_{6}^{(1)}}\left(-3 \Lambda_{0}\right)=\bigoplus_{s \in \mathbb{Z}} L_{E_{6}^{(1)}}\left(-3 \Lambda_{0}\right)^{(s)}, \quad L_{E_{6}^{(1)}}\left(-3 \Lambda_{0}\right)^{(s)}=\left\{v \in L_{E_{6}^{(1)}}\left(-3 \Lambda_{0}\right) \mid H(0) v=s v\right\}$.

The following proposition is analogous to Proposition 3.1;

Proposition 8.3. $L_{E_{6}^{(1)}}\left(-3 \Lambda_{0}\right)^{(0)}$ is a simple vertex subalgebra of $L_{E_{6}^{(1)}}\left(-3 \Lambda_{0}\right)$.

Each $L_{E_{6}^{(1)}}\left(-3 \Lambda_{0}\right)^{(s)}(s \in \mathbb{Z})$ is a simple $L_{E_{6}^{(1)}}\left(-3 \Lambda_{0}\right)^{(0)}$-module.

Clearly, $L_{E_{6}^{(1)}}\left(-3 \Lambda_{0}\right)^{(s)}$ is generated as $L_{E_{6}^{(1)}}\left(-3 \Lambda_{0}\right)^{(0)}$-module by $e_{(234)}(-1)^{s} \mathbf{1}$ for $s \in \mathbb{Z}_{\geq 0}$, and by $e_{\epsilon_{5}+\epsilon_{4}}(-1)^{-s} \mathbf{1}$ for $s \in \mathbb{Z}_{<0}$.

\section{Theorem 8.1.}

(a) We have:

$$
L_{E_{6}^{(1)}}\left(-3 \Lambda_{0}\right)^{(0)} \cong \widetilde{L}_{D_{5}^{(1)}}\left(-3 \Lambda_{0}\right) \otimes M(1) .
$$

In particular, $\widetilde{L}_{D_{5}^{(1)}}\left(-3 \Lambda_{0}\right)$ is a simple vertex operator algebra.

(b) Every $L_{E_{6}^{(1)}}\left(-3 \Lambda_{0}\right)^{(s)}(s \in \mathbb{Z})$ is an irreducible $L_{D_{5}^{(1)}}\left(-3 \Lambda_{0}\right) \otimes M(1)$ module. In particular

$$
\begin{aligned}
& L_{E_{6}^{(1)}}\left(-3 \Lambda_{0}\right)^{(s)} \cong L_{D_{5}^{(1)}}\left(-(s+3) \Lambda_{0}+s \Lambda_{4}\right) \otimes M(1, s) \quad(s \geq 0), \\
& L_{E_{6}^{(1)}}\left(-3 \Lambda_{0}\right)^{(s)} \cong L_{D_{5}^{(1)}}\left((s-3) \Lambda_{0}-s \Lambda_{5}\right) \otimes M(1, s) \quad(s<0) .
\end{aligned}
$$

Proof. We omit the proof because of its similarity with the proofs of Theorems 3.1 and 3.2. The main ingredients of the proof are the classification result from Proposition 8.2, the fusion rules argument (based on Proposition 7.2), formula for the conformal weight (8.9), and the fact that $L_{E_{6}^{(1)}}\left(-3 \Lambda_{0}\right)$ is simple vertex operator algebra.

We obtain the decomposition:

$$
\begin{gathered}
L_{E_{6}^{(1)}}\left(-3 \Lambda_{0}\right)=\bigoplus_{s \in \mathbb{Z}_{\geq 0}} L_{D_{5}^{(1)}}\left(-(s+3) \Lambda_{0}+s \Lambda_{4}\right) \otimes M(1, s) \\
\oplus \bigoplus_{s \in \mathbb{Z}_{<0}} L_{D_{5}^{(1)}}\left((s-3) \Lambda_{0}-s \Lambda_{5}\right) \otimes M(1, s) .
\end{gathered}
$$

As a consequence of decomposition (8.10), one also obtains the decomposition of $L_{F_{4}^{(1)}}\left(-3 \Lambda_{0}\right)$ into direct sum of $L_{B_{4}^{(1)}}\left(-3 \Lambda_{0}\right) \otimes M(1)^{+}$-modules, where $M(1)^{+}$denotes the $\mathbb{Z}_{2}$-orbifold of $M(1)$ (cf. [DN1, [DN2]). Namely, using 
conformal embeddings of $L_{F_{4}^{(1)}}\left(-3 \Lambda_{0}\right)$ into $L_{E_{6}^{(1)}}\left(-3 \Lambda_{0}\right)$ and $L_{B_{4}^{(1)}}\left(-3 \Lambda_{0}\right)$ into $L_{D_{5}^{(1)}}\left(-3 \Lambda_{0}\right)$ from [AP4], one can easily obtain that $L_{B_{4}^{(1)}}\left(-3 \Lambda_{0}\right) \otimes$ $M(1)^{+}$is a vertex subalgebra of $L_{F_{4}^{(1)}}\left(-3 \Lambda_{0}\right)$ with the same conformal vector.

We obtain the following decomposition:

Corollary 8.1. We have:

$$
\begin{gathered}
L_{F_{4}^{(1)}}\left(-3 \Lambda_{0}\right)=L_{B_{4}^{(1)}}\left(-3 \Lambda_{0}\right) \otimes M(1)^{+} \oplus L_{B_{4}^{(1)}}\left(-4 \Lambda_{0}+\Lambda_{1}\right) \otimes M(1)^{-} \\
\oplus \bigoplus_{s \in \mathbb{Z}_{>0}} L_{B_{4}^{(1)}}\left(-(s+3) \Lambda_{0}+s \Lambda_{4}\right) \otimes M(1, s) .
\end{gathered}
$$

\section{REFERENCES}

[A1] D. Adamović, Some rational vertex algebras, Glas. Mat. Ser. III 29 (49) (1994), 25-40.

[A2] D. Adamović, Representations of the vertex algebra $\mathcal{W}_{1+\infty}$ with a negative integer central charge, Comm. Algebra 29 (2001), 3153-3166.

[A3] D. Adamović, A construction of some ideals in affine vertex algebras, Int. J. Math. Math. Sci. (2003), 971-980.

[A4] D. Adamović, A construction of admissible $A_{1}^{(1)}$-modules of level -4/3, J. Pure Appl. Algebra 196 (2005), 119-134.

$[\mathrm{AM}]$ D. Adamović and A. Milas, Vertex operator algebras associated to modular invariant representations for $A_{1}^{(1)}$, Math. Res. Lett. 2 (1995), 563-575.

[AP1] D. Adamović and O. Perše, Representations of certain non-rational vertex operator algebras of affine type, J. Algebra 319 (2008), 2434-2450.

[AP2] D. Adamović and O. Perše, On coset vertex algebras with central charge 1, Math. Commun. 15 (2010), 143-157.

[AP3] D. Adamović and O. Perše, The vertex algebra $M(1)^{+}$and certain affine vertex algebras of level -1, SIGMA Symmetry Integrability Geom. Methods Appl. 8 (2012), Paper 040, 16 pp.

[AP4] D. Adamović and O. Perše, Some general results on conformal embeddings of affine vertex operator algebras, Algebr. Represent. Theory 16 (2013), 51-64.

[Ara] T. Arakawa, Rationality of admissible affine vertex algebras in the category $\mathcal{O}$, arXiv:1207.4857.

[B] R. E. Borcherds, Vertex algebras, Kac-Moody algebras, and the Monster, Proc. Natl. Acad. Sci. USA 83 (1986), 3068-3071.

[Bou] Bourbaki, Groupes et algèbras de Lie, Hermann, Paris, 1975.

[DM] C. Dong and G. Mason, On quantum Galois theory, Duke Math. J. 86 (1997), 305-321.

[DN1] C. Dong and K. Nagatomo, Classification of irreducible modules for the vertex operator algebra $M(1)^{+}$, J. Algebra 216 (1999), 384-404.

[DN2] C. Dong and K. Nagatomo, Classification of irreducible modules for the vertex operator algebra $M(1)^{+}$: II. Higher Rank , J. Algebra 240 (2001), 289-325.

$[\mathrm{FF}] \quad$ A. J. Feingold and I. B. Frenkel, Classical affine algebras, Adv. in Math. 56 (1985), 117-172.

[FB] E. Frenkel, D. Ben-Zvi, Vertex algebras and algebraic curves, Mathematical Surveys and Monographs, 88, American Mathematical Society, Providence, RI, 2001.

[FKRW] E. Frenkel, V. Kac, A. Radul and W. Wang, $\mathcal{W}_{1+\infty}$-algebra, $\mathcal{W}\left(\mathfrak{g l}_{N}\right)$-algebra with central charge N, Comm. Math. Phys. 2 (1995), 337-357. 
[FHL] I. Frenkel, Y.-Z. Huang, J. Lepowsky, On axiomatic approaches to vertex operator algebras and modules, Mem. Amer. Math. Soc. 104, 1993.

[FLM] I. Frenkel, J. Lepowsky, A. Meurman, Vertex Operator Algebras and the Monster, Pure and Appl. Math., Vol. 134, Academic Press, Boston, 1988.

[FZ] I. Frenkel and Y.-C. Zhu, Vertex operator algebras associated to representations of affine and Virasoro algebras, Duke Math. J. 66 (1992), 123-168.

$[\mathrm{H}] \quad$ J. E. Humphreys, Introduction to Lie algebras and representation theory, Graduate Texts in Mathematics, Vol. 9. Springer-Verlag, New York-Berlin, 1972. xii+169 pp.

[K1] V. G. Kac, Infinite dimensional Lie algebras, 3rd ed., Cambridge Univ. Press, Cambridge, 1990.

[K2] V. G. Kac, Vertex Algebras for Beginners, University Lecture Series, Second Edition, AMS, Vol. 10, 1998.

[Ko] B. Kostant, A formula for the multiplicities of the weight, Trans. Am. Math. Soc. 93 (1959), 53-73.

[KL] V. G. Kac and J. W. van de Leur, Super boson-fermion correspondence, Ann. Inst. Fourier (Grenoble) 37 (1987), 99-137.

[KR] V. G. Kac and A. Radul, Representation theory of the vertex algebra $\mathcal{W}_{1+\infty}$, Transform. Groups 1 (1996), 41-70.

[KW] V. Kac and M. Wakimoto, Integrable highest weight modules over affine superalgebras and Appell's function, Comm. Math. Phys. 215 (2001), 631-682.

[LL] J. Lepowsky, H. Li, Introduction to vertex operator algebras and their representations, Progress in Math., Vol. 227, Birkhauser, Boston, 2004.

[Li] H.-S. Li, Local systems of vertex operators, vertex superalgebras and modules. $J$. Pure Appl. Algebra 109 (1996), 143-195.

[L] A. Linshaw, Invariant theory and the $\mathcal{W}_{1+\infty}$ algebra with negative integral central charge, J. Eur. Math. Soc. (JEMS) 13 (2011), 1737-1768.

[MP] A. Meurman and M. Primc, Annihilating fields of standard modules of $s l(2, \mathbb{C})^{r}$ and combinatorial identities, Mem. Amer. Math. Soc. 137, AMS, Providence RI, 1999.

[O] S. Okada, Applications of Minor Summation Formulas to Rectangular-Shaped Representations of Classical Groups, J. Algebra 205 (1998), 337-367.

[P1] O. Perše, A note on representations of some affine vertex algebras of type $D$, to appear in Glas. Mat. Ser. III, arXiv:1205.3003.

[P2] O. Perše, Vertex operator algebras associated to type B affine Lie algebras on admissible half-integer levels, J. Algebra 307 (2007), 215-238.

[P3] O. Perše, Vertex operator algebras associated to certain admissible modules for affine Lie algebras of type A, Glas. Mat. Ser. III 43 (63) (2008), 41-57.

[W] W. Wang, $\mathcal{W}_{1+\infty}$-algebra, $\mathcal{W}_{3}$-algebra and Friedan-Martinec-Shenker bosonization, Comm. Math. Phys. 195 (1998), 95-111.

Faculty of Science - Department of Mathematics, University of Zagreb, Croatia

E-mail address: adamovic@math.hr; perse@math.hr 